\title{
O Emprego da Medicina Nuclear em Odontologia: o papel da cintilografia.
}

\section{The Use of Nuclear Medicine in Dentistry: the role of radionuclide imaging.}

\begin{abstract}
RESUMO
Medicina Nuclear constitui especialidade da Medicina que averigua a função de determinado órgão, empregando pequena quantidade de material radioativo designado por radiofármaco com finalidade diagnóstica e prognóstica, englobando tratamento de determinadas situações clínicas e de doenças. Manifestações de doenças sistêmicas podem repercutir na saúde bucal dos pacientes, nesse contexto de avaliação semiológica a Medicina Nuclear possui utilização na Odontologia como exame complementar. Sabe-se que o exame radiográfico odontológico apresenta determinada insuficiência na delimitação das imagens, o que requer muitas vezes o auxílio de outros recursos de diagnóstico por imagem para aprimoramento, dentre os quais os disponíveis com o emprego das técnicas de medicina nuclear, tal como com a utilização do recurso de técnica da cintilografia. O objetivo deste artigo foi o de realizar levantamento bibliográfico acerca dos possíveis usos da cintilografia em Odontologia. Realizou-se revisão bibliográfica com busca nas bases de dados Google Acadêmico e Literatura Latino-americana e do Caribe em Ciências da Saúde (LILACS) de estudos e artigos que tratavam do uso da medicina nuclear e da cintilografia em Odontologia. Em Odontologia a cintilografia óssea pode ser empregada no transcorrer das cirurgias bucais; na Ortodontia; durante a confecção e utilização dos aparelhos ortopédicos; na Implantodontia para averiguação da osseointegração nos implantes osseointegrados, dentre outras finalidades. O conhecimento e a conscientização acerca das técnicas de Medicina Nuclear e de cintilografia por parte do cirurgião dentista permite que ocorra emprego desses recursos sempre que for necessário o uso odontológico de um exame mais detalhado para confirmação da suspeita diagnóstica. A utilização da cintilografia em Odontologia mostra-se vantajosa e propicia a obtenção de vários benefícios em decorrência do seu uso, tais como aprimoramento diagnóstico e prognóstico.

Palavras-chave: Medicina Nuclear. Cintilografia. Diagnóstico. Odontologia.
\end{abstract}

\section{ABSTRACT}

Nuclear Medicine is specialty of medicine that investigates the function of certain organ, using a small amount of radioactive material designated as radiopharmaceutical for diagnostic and prognostic purposes, encompassing treatment of certain clinical situations and diseases. Manifestations of systemic diseases may have repercussions on the oral health of patients, in context of semiologic evaluation, Nuclear Medicine has use in Dentistry as a complementary examination. It is known that the dental radiographic examination presents a certain deficiency in the delimitation of the images, which often requires the help of other diagnostic imaging resources for improvement, among which are those available with the use of nuclear medicine techniques, such as with the use of the scintigraphy technique resource. The objective of this article was to perform a bibliographical survey about the possible uses of scintigraphy in dentistry. A bibliographic review was carried out with a search in the Latin American and Caribbean Health Sciences (LILACS) databases of studies and articles dealing with use of nuclear medicine and scintigraphy in dentistry in the databases Google Academic and Latin American and Caribbean Literature in Health Sciences. In Odontology the bone scintigraphy can be used in the course of the oral surgeries; in Orthodontics; during the manufacture and use of orthopedic appliances; in implant implants for osseointegration, among other purposes. The knowledge and awareness about the techniques of Nuclear Medicine and scintigraphy by the dental surgeon allows the use of these resources when the dental use of a more detailed examination is necessary to confirm the diagnostic suspicion. Use of scintigraphy in dentistry is advantageous and provides several benefits due to its use, such as diagnostic improvement and prognosis. Keywords: Nuclear Medicine. Radionuclide Imaging. Diagnosis. Dentistry.

\begin{abstract}
Sérgio Spezzia ${ }^{1}$
${ }^{1}$ Cirurgião Dentista. Especialista em Saúde da Mulher no Climatério pela Faculdade de Saúde Pública da Universidade de São Paulo. Especialista em Gestão Pública pela Universidade Tecnológica Federal do Paraná. Especialista em Gestão em Saúde pela Universidade Federal de São Paulo. Especialista em Adolescência para Equipe Multidisciplinar e Mestre em Ciências pela Escola Paulista de Medicina - Universidade Federal de São Paulo.
\end{abstract}

Endereço para Correspondência:

Sérgio Spezzia

Rua Silva Bueno, 1001, Ipiranga,

CEP 04208-050, São Paulo, SP

sergio.spezzia@unifesp.br 


\section{INTRODUÇÃO}

A imagem médica consta de procedimento executado com finalidade de levantar imagens de um organismo para análise. Nesse contexto, a Medicina Nuclear consiste em uma das técnicas de maior emprego ${ }^{1}$.

A Medicina Nuclear constitui uma especialidade da Medicina que averigua a função de determinado órgão, empregando pequena quantidade de material radioativo designado por radiofármaco ou por traçadores moleculares com finalidade diagnóstica e prognóstica, englobando tratamento de determinadas situações clínicas e de doenças. Nesse processo ocorre detecção da radiação gama oriunda dos radioisótopos. Essa técnica possibilita avaliar a performance metabólica dos pacientes. A dose utilizada para exame pode usar fontes radioativas dentro ou fora do organismo, respectivamente com emprego da exposição interna ou externa ${ }^{2,3}$.

O emprego da Medicina Nuclear agrega estudos planares, que podem ser estático ou dinâmicos e estudos tomográficos. Na categoria dos estudos planares têm-se por exemplo a cintilografia óssea e a cintilografia das glândulas salivares que tem aplicabilidade em Odontologia ${ }^{4}$.

Manifestações de doenças sistêmicas podem repercutir na saúde bucal dos pacientes, nesse contexto de avaliação semiológica a Medicina Nuclear possui utilização na Odontologia como exame complementar ${ }^{5,6}$.

A determinação diagnóstica possui papel fundamental para que ocorra escolha adequada do tipo de tratamento que será realizado. Em Odontologia convive-se com determinadas situações em que necessita-se empregar diversos recursos clínicos e radiográficos na análise semiótica para elucidação diagnóstica ${ }^{5,6}$.

Sabe-se que ocorreu o aprimoramento de técnicas de obtenção por imagem na Odontologia e que dentre as técnicas disponíveis, têm-se a possibilidade de uso da Medicina Nuclear e da cintilografia, que atuam aperfeiçoando a obtenção das imagens acerca de lesões ${ }^{5,7-9}$.

Exames de Medicina Nuclear podem ser utilizados, visando detecção de processos patológicos endodônticos e/ou periodontais, bem como podem ser empregados na Endodontia, no intuito de obter diagnóstico precoce e a localização correta dos focos infeccio$\operatorname{sos}^{10-12}$.

Na Odontologia em Endodontia existe dificuldade na determinação diagnóstica a nível periapical, uma vez que pode haver divergências entre os resultados clínicos e histológicos obtidos. Alterações periapicais costumeiramente empregam para análise exames radiográficos obtidos com métodos estáticos e bidimensionais, o que mostra-se insuficiente para averiguar alterações periapicais em fases iniciais. Alterações periapicais e periodontais normalmente são visualizadas apenas depois de decorrido determinado período do seu início. Ocorre que a percepção das modificações na estrutura do tecido mineralizado depende da sensibilidade do exame radiográfico empregado, comumente observa-se perda óssea menor do que procede normalmente. O exame radiográfico odontológico, portanto, apresenta determinada insuficiência na delimitação das imagens, o que requer o auxílio de outros recursos de diagnóstico por imagem para complemento, dentre os quais a cintilografia $^{10-12,13}$. 
O objetivo deste artigo foi o de realizar levantamento bibliográfico acerca dos possíveis usos da cintilografia em Odontologia.

\section{MÉTODO}

Realizou-se revisão bibliográfica com busca nas bases de dados Google Acadêmico e Literatura Latino-americana e do Caribe em Ciências da Saúde (LILACS) de estudos e artigos que tratavam do uso da medicina nuclear e da cintilografia em Odontologia. No Google Acadêmico a expressão de busca empregada foi cintilografia and medicina nuclear and odontologia and diagnóstico and saúde bucal e encontrou-se aproximadamente 263 resultados. No LILACS utilizou-se a expressão de busca medicina nuclear and cintilografia and odontologia e obteve-se 22 registros.

Incluiu-se artigos publicados nos idiomas inglês e português em periódicos nacionais e internacionais.

Excluiu-se artigos que não possuíam conteúdo concernente com o pesquisado após realização de leitura seletiva e analítica de todos os artigos levantados.

Apontamentos de livros, trabalhos, monografias, dissertações e teses sobre a mesma temática de pesquisa também foram incluídos.

\section{REVISÃO DE LITERATURA}

O exame clínico odontológico e os exames complementares são extremamente importantes na elaboração do diagnóstico, nesse contexto, a imagem representa um recurso diagnóstico que possui papel de destaque ${ }^{14,15}$.

De acordo com Meneguin et al., (2002) ${ }^{16}$ nos atendimentos odontológicos em geral, averiguou-se ocorrer maior realização de procedimentos com cunho tanto preventivo como curativo, o que ocasionou maior longevidade dos dentes em boca, estes que exigiram tratamentos especializados para manutenção da sua saúde, dessa forma exames complementares tornaram-se elementares para aprimorar o planejamento terapêutico, entre eles o recurso da utilização da imagem mostrou-se favorável no diagnóstico odontológico. Alterações nas estruturas ósseas que envolvem esses elementos dentais podem ser melhor visualizados empregando-se por exemplo, a cintilografia.

Na cintilografia ocorre detecção pela câmara de cintilação da radiação gama emitida pelo radiofármaco ou radioisótopo, como por exemplo, tecnécio-99m, iodo-131, gálio-67, índio-111, que foi injetado por via endovenosa na corrente sanguínea do paciente anteriormente, permitindo a obtenção de imagens via processo físico; na sequência passado determinado período a visualização das imagens é possível em um receptor tal como um monitor de computador. A imagem obtida pode ocorrer com uso de detector em movimento com imagem por varredura ou pode ocorrer com utilização de um detector imóvel com imagem estacionária. As técnicas preconizadas usam compostos fosfatados, possibilitando desfechos favoráveis nos levantamentos cintilográficos ósseos ${ }^{13,17,18}$.

A utilização da cintilografia óssea possibilita na área médica a identificação de doenças e de prováveis alterações metabólicas, pode-se evidenciar características do metabolismo normal e das modificações de caráter fisiológico. As indicações de uso da 
cintilografia envolvem alterações vasculares e ósseas, traumas e patologias inflamatórias, neoplásicas e metabólicas ${ }^{18}$.

A cintilografia óssea trifásica (COT) comumente é utilizada em Medicina Nuclear, uma vez que consta de técnica sensível, pouco onerosa e que fornece imagens de forma rápida ${ }^{18}$.

Em conformidade com Brooks, $(2005)^{19}$, a COT apresenta sensibilidade satisfatória, sendo capaz de evidenciar lesões existentes que ainda não são passíveis de averiguação em exames radiográficos tradicionais.

A análise do metabolismo ósseo, averiguando-se desordens do desenvolvimento pode ocorrer fazendo-se uso da cintilografia, principalmente na área maxilofacial. A técnica da COT pode ser empregada na averiguação das deformidades crânio-maxilo-faciais, por intermédio dela pode-se realizar a mensuração do crescimento e pode-se evidenciar dados acerca da formação e remodelação óssea ${ }^{18,20,21}$.

A cintilografia possibilita averiguação das características funcionais e fisiológicas da articulação temporomandibular e é utilizada para verificar precocemente alterações de caráter inflamatório que podem instalar-se. O exame permite identificar os radiofármacos que tem atração por leucócitos ${ }^{22,23}$.

Em Odontologia a cintilografia óssea pode ser empregada no transcorrer das cirurgias bucais; na Ortodontia; durante a confecção e utilização dos aparelhos ortopédicos; na Implantodontia para averiguação da osseointegração nos implantes osseointegrados, dentre outras finalidades. Essa técnica possibilita a avaliação do crescimento facial e permite averiguar a performance fisiológica do crescimento ósseo ${ }^{24-26}$.

Na Ortodontia é de fundamental importância analisar-se o crescimento e desenvolvimento craniofaciais dos pacientes, somente dessa forma torna-se viável a elaboração do planejamento terapêutico. Informações obtidas acerca dessas manifestações de crescimento e desenvolvimento de forma adequada possibilitam aperfeiçoamento do plano de tratamento ortodôntico a ser elaborado. A cintilografia óssea constitui uma técnica dinâmica para verificação do crescimento craniofacial, que mostra-se bastante útil, principalmente nas fases de diagnóstico e de planejamento odontológico ${ }^{27-31}$.

$\mathrm{Na}$ área da saúde, inclusive na Odontologia existe interesse voltado para o aperfeiçoamento diagnóstico e para o aprimoramento dos tratamentos e planejamentos terapêuticos a serem adotados. Nesse contexto, busca-se a utilização dos recursos disponíveis pelo avanço tecnológico, entre os quais os recursos para obtenção de imagens novos, como o emprego da Medicina Nuclear ${ }^{1-3,5,6}$.

\section{DISCUSS ÃO}

O diagnóstico por imagem depende da análise dos sinais presentes nos exames de imagem para ocorrer a elaboração do diagnóstico definitivo das doenças, entretanto, é imprescindível que ocorra disponibilidade de parâmetros suficientemente capazes para determinação exata das doenças, como o recurso da Medicina Nuclear ${ }^{1-3,5,6}$.

De acordo com Guedes, $(1987)^{32}$, existem na Odontologia tecnologias novas disponíveis, entre as quais recursos que permitem diagnósticos por imagem aprimorados.

A utilização do método de cintilografia óssea permite a obtenção de algumas vantagens, uma vez que essa técnica possui alta especificidade, boa sensibilidade e pequena 
agressividade ao ser aplicada ${ }^{33-35}$.

O uso da Medicina Nuclear pode obter êxito ao ser empregada, quando não for viável obter imagens por outros recursos diagnósticos, nessas circunstâncias pode-se optar pelo uso odontológico da cintilografia ${ }^{5}$.

No transcorrer da realização dos exames de Medicina Nuclear são necessárias algumas medidas de proteção aos profissionais e pacientes, como o estabelecimento da dose efetiva anual. O cálculo da dose aplicada nos pacientes que receberam radionuclídeos é dificultoso, ao passo que ocorre distribuição diferenciada entre as regiões do corpo. Existem ainda outros fatores que influem na realização do cálculo da dose a ser aplicada, tais como: quantidade de energia liberada; tipo de radiação escolhido para uso; meia vida física e biológica ${ }^{36-38}$.

Mulheres em fase de gravidez ou lactação não devem receber radionuclídeos, uma vez que a realização de exames de Medicina Nuclear nesses estágios da vida das mulheres podem repercutir desfavoravelmente, ocorrendo irradiação do feto em grávidas e possível aparecimento de radioatividade no leite em mulheres que amamentam, conforme o radiofármaco que foi escolhido para uso $^{36}$.

Existe uma diferença significativa na forma como procede a aplicação dos exames de Medicina Nuclear na rede pública e privada de assistência à saúde. Em âmbito público, o Sistema Único de Saúde (SUS) preconiza o seu uso, mas com restrições. Muitos procedimentos que figuram como eletivos no SUS, dificilmente são executados ${ }^{39,40}$.

Convém ressaltar que o SUS convive com restrição de recursos para determinados procedimentos, que são classificados como sendo de alta complexidade e de custos elevados, dentre os quais insere-se os de Medicina Nuclear ${ }^{39,40}$.

O conhecimento e a conscientização acerca das técnicas de Medicina Nuclear e de cintilografia por parte do cirurgião dentista permite que ocorra emprego desses recursos sempre que for necessário o uso odontológico de um exame mais detalhado para confirmação da suspeita diagnóstica.

\section{CONCLUSÕES}

- A utilização da cintilografia em Odontologia mostra-se vantajosa e propicia a obtenção de vários benefícios em decorrência do seu uso, tais como aprimoramento diagnóstico e prognóstico.

- A relação custo-benefício acerca do seu emprego mostra-se favorável, uma vez que ocorrem benefícios clínicos que permitem a elaboração de planos de tratamento odontológicos melhor delineados.

\section{REFERENCIAS}

1. Caetano R. Inovações e trajetórias tecnológicas no território das imagens médicas. [Tese]. Rio de Janeiro: Instituto de Medicina Social - Universidade do Estado do Rio de Janeiro, 2002.

2. Thrall J, Ziessman H. Nuclear Medicine - The Requisits, 2nd ed, Mosby, St Louis, 2001.

3. de Lima JMP, Lapa P. Medicina nuclear. cap. 3, p. 1-10. In: Lima JMP. Imagiologia Básica - Diferentes Técnicas de Imagiologia. Lidel Eds Técnicas, 2009. Disponível em: http://rihuc.huc.min-saude.pt/ bitstream/10400.4/977/1/000-CAP03\%2010\%20p.pdf Acessado em 08 de março de 2019. 
4. Reis ARR. Aquisição, Processamento e Análise de Imagens de Medicina Nuclear. [Dissertação]. Mestrado em Engenharia Biomédica e Biofísica Engenharia Clínica e Instrumentação Médica. Lisboa: Universidade de Lisboa, 2012.

5. Freire ARS, Lima ENP, Almeida OP, Kowalski LP. Medicina nuclear na odontologia. Rev Assoc Paul Cir Dent, 2002; 56(6):447-51.

6. Lima JP. Radiologia e Medicina Nuclear. Acta Radiol Portug, 2014, 26(102):7.

7. Souza LC, Davidowicz H, Wagner J. Análise comparativa de imagens hipercaptantes em maxila e mandíbula, frente ao mapeamento cintilográfico dos ossos da face e o mapeamento cintilográfico dos ossos da face com leucócitos marcados. Rev Inst Ciênc Saúde, 2003; 21(3):249-54.

8. Walivaara DA, Abrahamsson P, Isakssom S, Blomqvist JE, Samfors KA. Prospective study of periapically infected teeth treated with periapical urgery including ultrasonic preparation and restorative material root-end fillings. J Oral Maxillofac Surg, 2007; 65(5):931-5.

9. Cristofer ACS, Bolognesi L. Aplicações Terapêuticas em Medicina Nuclear. Tekhne e Logos, 2014; 5(2):153-67. 10. Shimamoto H, Tatsumi M, Kakimoto N, Hamada S, Shimosegawa E, Murakami S et al. (18)F-FDG accumulation in the oral cavity is associated with periodontal disease and apical periodontitis: an initial demonstration on PET/CT. Ann Nucl Med, 2008; 22(7):587-93.

11. Carvalho ALP. Avaliação da correlação de imagens da tomografia por emissão de pósitrons (PET-fluoreto), com as alterações perirradiculares da cavidade oral. [Dissertação]. São Paulo: Faculdade de Odontologia da Universidade Paulista, 2010.

12. Spezzia S. A Tomografia por Emissão de Pósitrons (PET) como Recurso Diagnóstico em Endodontia. In: VIII Congresso Internacional de Odontologia UNIBAN/Anhanguera, 2012, São Paulo - SP. Anais do VIII Congresso Internacional de Odontologia UNIBAN/Anhanguera, 2012. p. 86.

13. Corey KR, Kenny P, Greenber E, Pazianos A, Pearson OH, Laughlin JS. The use of calcium 47 in diagnostic studies of patients with bone lesions. Am j Roentgenol, 1961; 85:955-75.

14. Gil C, Oliveira JX, Cavalcanti MGP. Avaliação da interação de algumas técnicas radiográficas com as diferentes especialidades odontológicas. Rev Odontol UNICID, 1999; (11):25-38.

15. Vieira EMM, Coclete GA, Salzedas LMP, Castro IO, Demathé A. Inter-relação cirurgião dentista e radiologista: sucesso no diagnóstico odontológico. Rev Odonto Metodista, 2008; 16(32):25-30.

16. Meneguim MC, Pereira AC, Silva FRB. Prevalência de Cárie Radicular e Condição Periodontal em uma População Idosa Institucionalizada de Piracicaba. São Paulo, Pesq Odont Bras, 2002; 16(1):50-6.

17. Bergestedt HF. Bone scintigraphy of facial skeleton with 99Tcmdiphosphonate. Acta Radiol Diagn, 1975; 16(4):337-41.

18. Amorim BJ. SBMN - Sociedade Brasileira de Medicina Nuclear. Guideline para Cintilografia Óssea e Cintilografia Óssea Trifásica, 2018. Disponível em: http://sbmn.org.br/wp-content/uploads/2018/08/Guideline-de-Cintilografia-\%C3\%93ssea.pdf Acessado em 07 de março de 2019.

19. Brooks ME. The skeletal system. In: Sharp, PF, Gemmell HG, Murray AD. Practical nuclear medicine. 3. ed. London: Springer-Verlag; 2005. p.149-62.

20. Kaban LB. Skeletal Scintigraphy for Assessment of mandibular Growth and Asymmetry. Pediatric Nuclear Medicine. New York: Springer Verlag; 1995.

21. Nishimura T, Iizuka T. Evaluation of the pathophysiology of odontogenic maxillary sinusitis using bone scintigraphy. Int J Oral Maxillofac Surg, 2002; 31:389-96.

22. Vasconcelos BCE. Meios de Diagnóstico das Desordens Têmporomandibulares. Rev Cir Traumat Buco-Maxilo-Facial, 2002; 1(2):49-57.

23. Brasileiro CB, Cardoso VN, Ruckert B, Campos TPR. Avaliação de processos inflamatórios na articulação têmporomandibular empregando leucócitos autólogos marcados com tecnécio-99m em modelo animal. Radiol Bras, 2006; 39(4):283-6.

24. Morelli CG, Davidowicz H, Moura AAM. Cintilografia x ressonância magnética: revisão da literatura. Rev Inst Ciênc Saúde, 1996; 14:81-5.

25. Paulsen HU, Rabol A, Sorensen SS. Bone scintigraphy of human temporomandibular joints during Herbst treatment: a case report. Eur J Orthod, 1998; 20:369-74.

26. Hodder SC, Rees JI, Oliver TB, Facey PE, Sugar AW. SPECT bone scintigraphy in the diagnosis and management of mandibular condylar hyperplasia. Br J Oral Maxillofac Surg, 2000; 38:87-93.

27. Green LJ. The interrelationships among height, weight, and chronological, dental and skeletal ages. Angle Orthod, 1961; 31(3):189-93.

28. Grave KC, Brown T. Skeletal ossification and adolescent growth spurt. Am J Orthod Dentofacial Orthop, 1976; 69(6):611-9. 
29. Hägg U, Taranger J. Maturation indicators and the puberal growth spurt. Am J Orthod, 1982; 82(4):299-309. 30. Moore RN, Moyer BA, DuBois LM. Skeletal maturation and craniofacial growth. Am J Orthod Dentofacial Orthop, 1990; 98(1):33-40.

31. Gomes AS, Lima EM. Mandibular growth during adolescence. Angle Orthod, 2006; 76(5):786-90.

32. Guedes AML. Interpretação radiográfica sistemática de laudo computadorizado. [Tese de Livre-docência]. Rio de Janeiro: Faculdade de Odontologia - Universidade do Estado do Rio de Janeiro, 1987.

33. Cisneros GJ, Kaban LB. Computerized skeletal scintigraphy for assessment of mandibular asymmetry. J Oral Maxillofac Surg, 1984; 42:513-20.

34. Murray IPC. Bone scintigraphy: the procedure and interpretation. In: Murray IPC, Ell RJ, eds. Nuclear medicine in clinical diagnosis and treatment. Edinburgh: Churchill Livingstone, 1994.

35. Sapienza MT. Medicina Nuclear - princípios e aplicações. 2. ed, Ed Atheneu, 2017.

36. Dendy PP, Goldstone KE, Parkin A, Barber RW. Radiation Protection. In: Sharp, PF, Gemmell HG, Murray AD. Practical nuclear medicine. 3. ed. London: Springer-Verlag; 2005. p. 149-62.

37. Brasil. Ministério da Ciência, Tecnologia e Inovação. Comissão Nacional de Energia Nuclear. CNEN NN 3.05 - Requisitos de radioproteção e segurança para serviços de medicina nuclear. [acessado em 10 de março de 2013]. Disponível em: http://www.cnen.gov.br/seguranca/normas/pdf/Nrm305.pdf.

38. Santos LAN. Estudo dos Aspectos Radiográficos e Cintilográficos da Displasia Fibrosa. [Tese]. Piracicaba: Faculdade de Odontologia de Piracicaba, da Universidade Estadual de Campinas, 2011.

39. Pozzo L, Coura Filho G, Osso Júnior JA, Squair PL. O SUS na medicina nuclear do Brasil: avaliação e comparação dos dados fornecidos pelo Datasus e CNEN. Radiol Bras, 2014; 47(3):141-8.

40. Tinoco C. Sociedade Brasileira de Medicina Nuclear. Plano Nacional de Expansão da Medicina Nuclear no SUS, 2015. Disponível em: http://sbmn.org.br/wp-content/uploads/2016/01/Workshop_ClaudioTinoco_Por-que-expans\%C3\%A3o-da-Medicina-Nuclear.pdf Acessado em 08 de março de 2019. 